\title{
Comparing land-cover maps accuracies generated from multispectral classification of Landsat-8 OLI dan Pleiades images using two different classification schemes
}

Erisa Ayu W. Putri, Projo Danoedoro, Nur Farda

Erisa Ayu W. A. Putri, Projo Danoedoro, Nur M. Farda, "Comparing landcover maps accuracies generated from multispectral classification of Landsat-8 OLI dan Pleiades images using two different classification schemes," Proc. SPIE 11311, Sixth Geoinformation Science Symposium, $113110 U$ (21 November 2019); doi: 10.1117/12.2548888

Event: Sixth Geoinformation Science Symposium, 2019, Yogyakarta, Indonesia 


\title{
Comparing Land-cover Maps Accuracies Generated from Multispectral Classification of Landsat-8 OLI dan Pleiades Images using Two Different Classification Schemes
}

\author{
Erisa A. W. Putri ${ }^{1, \mathrm{a}}$, Projo Danoedoro ${ }^{2, \mathrm{~b}}$, Nur M. Farda, ${ }^{3 \mathrm{c}}$

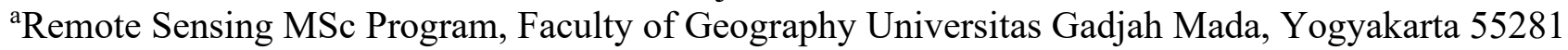 \\ ${ }^{\mathrm{b}}$ Remote Sensing Laboratory, Faculty of Geography Universitas Gadjah Mada, Yogyakarta 55281 \\ ${ }^{\mathrm{c}}$ Geographical Information System Laboratory, Faculty of Geography Universitas Gadjah Mada, \\ Yogyakarta 55281
}

\begin{abstract}
Multispectral classification is one of the main methods in the analysis and processing of digital remotely sensed imagery, which until now is still widely used to generate land-cover/ land-use information. Technically, pixel-based classification methods rely on conventional approaches, as compared to GeoBIA, and it can be implemented using either supervised or unsupervised methods. The classification methods are supported by the rapid development of various image processing software, which provide a wide variety of algorithm options, so that the classification process can be carried out easily. Although relatively simple, an appropriate selection of multispectral classification algorithm may provide highly accurate land-cover maps. However, the highly accurate land-cover/land-use maps may also be influenced by image types and classification schemes that are used in the study. This study aimed to compare the results of the multispectral classification using maximum likelihood algorithm, for generating land-cover maps based on Landsat-8 OLI images (30 meters) and Pleiades imagery ( 2 meters). The classification referred to two different classification schemes relating to spectral and spatial dimensions. The results showed that the multispectral classification with spectral-related classification scheme applied to Pleiades imagery gave higher overall accuracy as compared to that of Landsat-8 OLI. It was also found that the highest overall accuracy achieved in this study was $81.7 \%$, obtained using Pleiades imagery and referring to spectral dimension classification scheme. On the other hand, the lowest overall accuracy was obtained by the same imagery applied using spatial-related dimension. The relatively similar values of low overall accuracy for spatial-related dimension was also gained by Landsat-8 OLI imagery, proving that multispectral classification does not work well for spatial-related land cover classification scheme.
\end{abstract}

Keywords: multispectral classification, classification scheme, spectral dimension, spatial dimension, Landsat-8 OLI, Pleiades, accuracy assessment

\section{INTRODUCTION}

Land cover is one of the important information that is naturally dynamic and widely needed as the basis for various activities related to development planning, natural resource management, as well as spatial modeling of human interaction with the environment. Considering the importance of its information for various parties and domains, the land cover inventory needs to be carried out effectively and efficiently. Remote sensing is a proven technology which could be employed for generating land cover data effectively and efficiently [1], with no direct contact needed with the corresponding objects. The usage of such technology only requires validation of interpretation results or generally mentioned as a ground check to produce information that can be scientifically accountable and acceptable.

Digital image analysis is one of the various methods for generating land cover information, which has been rapidly developing along with the vast development of remote sensor technology. Multispectral classification is a basic function in remotely sensed digital image analysis [2] which classifies digital numbers (DNs) as a representation of various land-

\footnotetext{
${ }^{1}$ erisawaspadi@gmail.com

2 projo.danoedoro@geo.ugm.ac.id (corresponding author)

3 farda@geo.ugm.ac.id
} 
cover objects in the Earth's surface. Although there are tremendous advances in digital image analysis techniques, multispectral classification is still widely used to generate land cover maps due to the simplicity of its operation. The ease of multispectral classification usage is supported by the availability of various digital image processing applications containing numerous classification algorithm options. The usage of appropriate classification algorithms concerning the data's characteristics possibly produce highly accurate of land cover maps. Moreover, Estoque, et al., (2015) states that several other triggering factors resulting in highly accurate maps are the classification scheme being used [2]. The classification schemes appear as the framework influencing the image selection, the opted method, and the detailed information needed in the land cover map production.

This study aimed to compare the results of the usage of multispectral classification for generating land-cover maps based on Landsat-8 OLI images (30 meters) and Pleiades imagery (2 meters) within two different classification schemes. The classification schemes used in this study were the spectral and spatial-related land cover proposed by [3] and [4]. Those two dimensions of classification schemes were selected with respect to the land cover types found in the study area, as listed in Table 1.

Table 1. Selected classification scheme, modified from [3] and [4]

\begin{tabular}{llll}
\hline Spectral related cover dimension & \multicolumn{2}{l}{ Spatial dimension } \\
Code & Class object & Code & Class object \\
C11 & Deep water & S11 & Sea \\
C12 & Shallow water & $S 12$ & Lake \\
C22 & Woody broadleaves & $S 13$ & River and channel \\
C22 & Non-woody broadleaves & $S 21$ & Block coverage vegetation \\
C31 & Bare soil, dry & $S 22$ & Field pattern \\
C32 & Bare soil, moist & $S 24$ & Other vegetation feature with specific shapes \\
C41 & Asphalt, concrete and & $S 31$ & Coastal barren land \\
& cemented surface & $S 41$ & Built -up area, whether isolated or not \\
C42 & Compacted clay surface & $S 43$ & Built-up features with linear shapes \\
\hline
\end{tabular}

\section{METHODS}

\subsection{Study area and dataset}

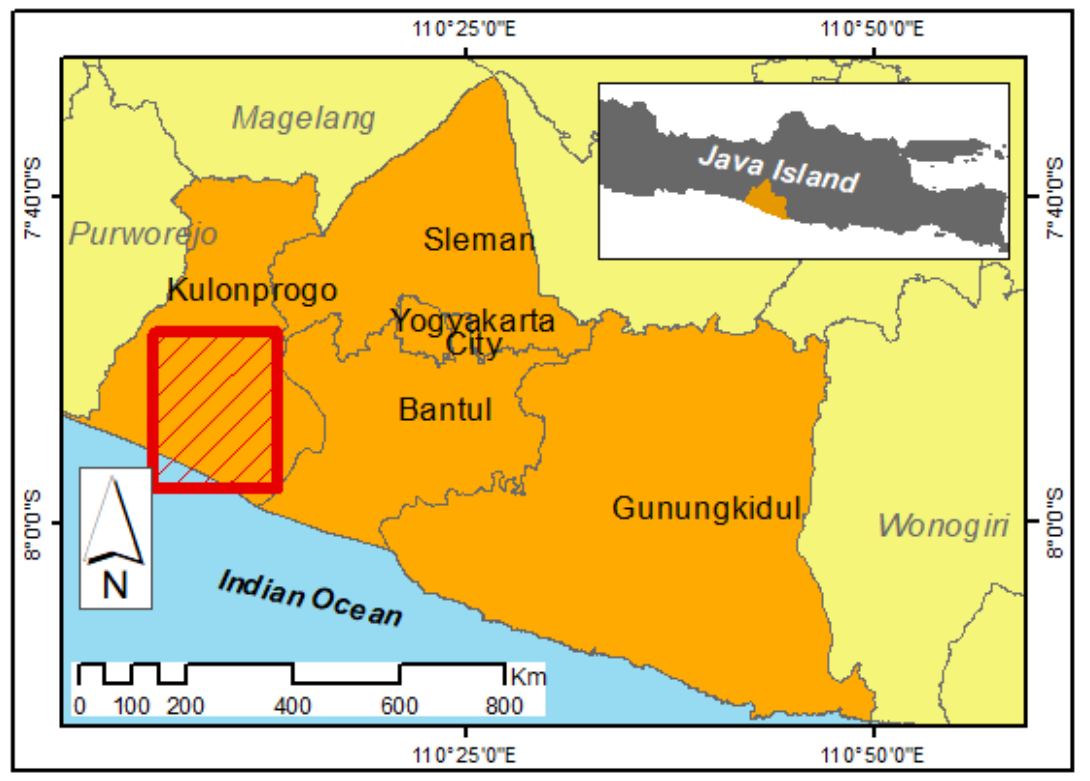

Figure 1. Part of Kulonprogo district, which was selected as the study area

As shown in the Figure 1, this study made use of all multispectral reflective bands of Landsat-8 OLI and Pleiades recorded on May 05, 2018 and April 27, 2018, respectively. The significant difference of spatial resolution as well as spectral in 
Landsat-8 OLI (7 multispectral bands with 30-m pixel size) and Pleiades (4 multispectral bands with 2-m pixel size) were compared to determine the effect of both resolution on the spectral and spatial classification scheme dimension using multispectral classification technique. For these two datasets, a relative radiometric correction using DOS (Dark-objectsubtraction) [5] and cloud masking were performed to normalize the distribution of pixel value in each imagery. Even though no absolute necessities to actuate the relative radiometric correction [5], it would improve the spectral aspect and visual appearance which would ease the training sample selection and analysis by the interpreter.

The DOS's corrected and cloud-free imagery was then cropped in order to obtain Kulonprogo District as the area of interest, and to ease the classification execution as well as ground truth checking. The selection of the study area as showed in Figure 1 was based on the variation of land cover types in the southern part of Kulonprogo. In the study area, there were various features such as water bodies features (namely Sermo reservoir, Progo river, and part of Indian ocean), vegetation (both dense and thin/sparse) cover, built-up areas, barren land, cultivated land, and many more. The existence of almost all classes of land cover features in each referred classification schemes was expected to provide a comprehensive understanding of the comparison of classification schemes and methods for the two images used in this study.

\subsection{Algorithm selection}

Multispectral classification could be done either with a supervised or unsupervised approach using a variety classification algorithm. The selection of algorithm becomes an essential part of multispectral classification which directly affects the statistical tendency of the pixel to be labeled as a particular class of objects. The use of different algorithms in the supervised classification applied for the same training sample possibly would produce land cover maps with different rates of accuracy. Therefore, the selection of algorithm in the multispectral classification is needed to be based on the understanding of the calculation process behind the algorithm utilized.

Maximum likelihood is one of the most widely used algorithms in the classification of digital imagery or in remote sensing field $[6,7,8]$. It was considered to be the most statistically stable algorithm to be applied in remotely sensed image analysis and one of the most accurate classifiers due to the usage of various statistical features in its process [7,9]. Technically, maximum likelihood classifies pixel values based on probability calculation represented on the shape, size, and orientation of the sampled feature space [8]. The assumption deployed in the classifier was the homogeneous object will always display a normal distribution (Gaussian) histogram where the distribution of the sample's spectral signature could be fully described using mean vectors and covariance matrices [9].

\subsection{Classification procedures}

Supervised classification using maximum likelihood was applied on the cropped Landsat-8 OLI and Pleiades imagery to produce spectral and spatial-related land cover maps. Through the classification method, less than 100 pixels were taken either in the form of points or polygons are proportionally taken to be calculated as a training sample. Point as a retrieval sample unit was carried out on Landsat-8 OLI imagery for training C41, S13 and S42 classes which have elongated patterns and narrow dimension compared to Landsat-8 OLI's spatial resolution; whereas the rest of class features in that imagery as well as that of Pleiades were taken in the form of polygons. The two kinds of retrieval sample unit were applied as a strategy to minimize the possibility of accidentally mixed-pixel taken which would decrease its separability indices, considering the low spatial resolution of Landsat-8 OLI.

Technically, the training sample was retrieved in several tentative feature classes to facilitate the computer calculation in distinguishing several spectral variations of the same class object either in spatial or spectral dimensions classification schemes. The tentative classes were then merged into the main class features for both classification schemes (Table 1) in the post-classification stage, to be calculated for its classification accuracy results. In that stage, a $3 \times 3$ pixels of majority filter was selectively applied to the whole feature class but C11, C4, S13, and S43, which having elongated pattern and relatively narrow dimension. The selective filtering was needed to minimize salt-and-pepper effect which frequently found in multispectral classification results as well as to fulfill the MMU (Minimum mapping unit) referring to [10].

\subsection{Accuracy assessment}

An area-based accuracy assessment using an error matrix [11] was performed for each classification results to determine the most accurate land cover map produced in this study. A total of 81 sample areas collected from field-work were selected as the basic calculation of overall accuracy as well as producer's and user's accuracy of each feature class. The sample was stratified-randomly selected considering the stratified land cover class, related to the spectral and spatial dimensions, and also to the proportion of coverage area for each feature objects. The usage of area (or polygon)-based instead of point- 
based samples in the error matrix calculation was conducted to minimize the possibility of low accuracy results due to the binary decision used in the point-based calculation.

\section{RESULTS AND DISCUSSION}

\subsection{Spectral-related land cover classification scheme}

The results of spectral-related classification scheme using Landsat-8 OLI and Pleiades imagery were shown in figure 1a and $1 \mathrm{~b}$ respectively. The spatial resolution differences between those images were considerably giving a significant impact on the detail of classification results, especially for narrow objects with elongated patterns as rivers, waterways, and roads which were categorized as C12 and C41. By using Landsat-8 OLI, the representative appearance of C12 was only found in the related object having enough dimension (larger than the one-pixel size of Landsat-8 OLI) like Sermo reservoir located in the northwest of the study area (upper left of the images). On the other hand, the classes with specific patterns were able to be well classified using Pleiades imagery.

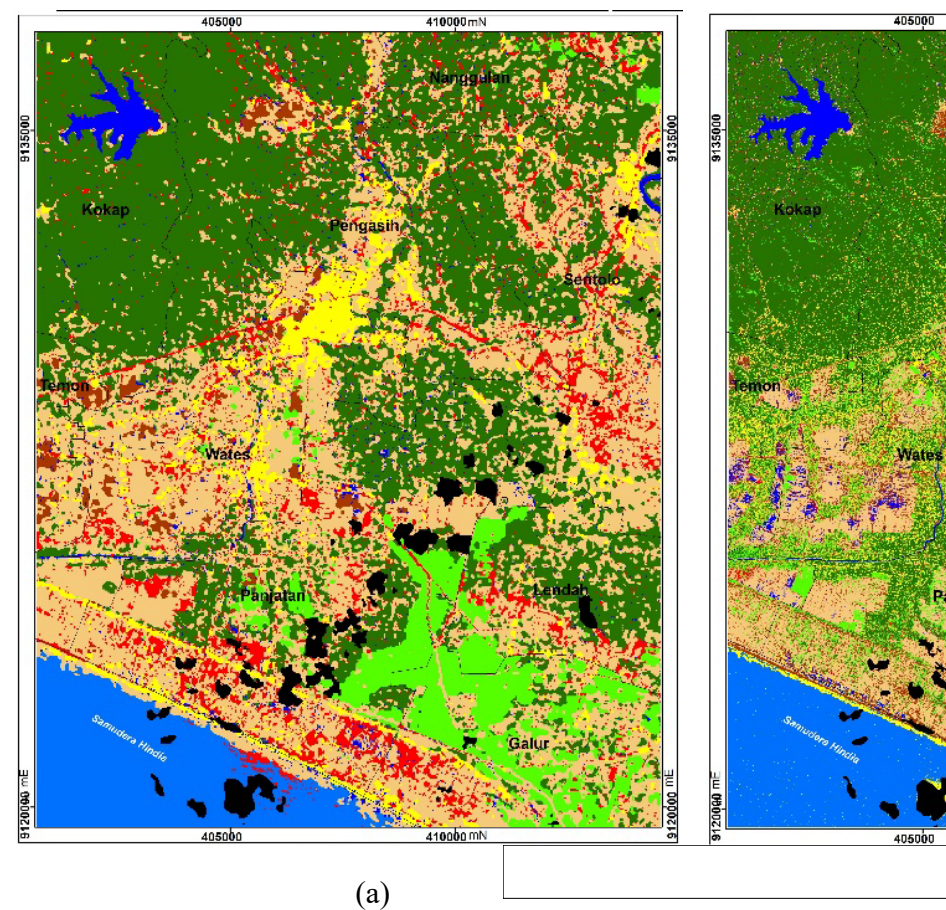

(a)

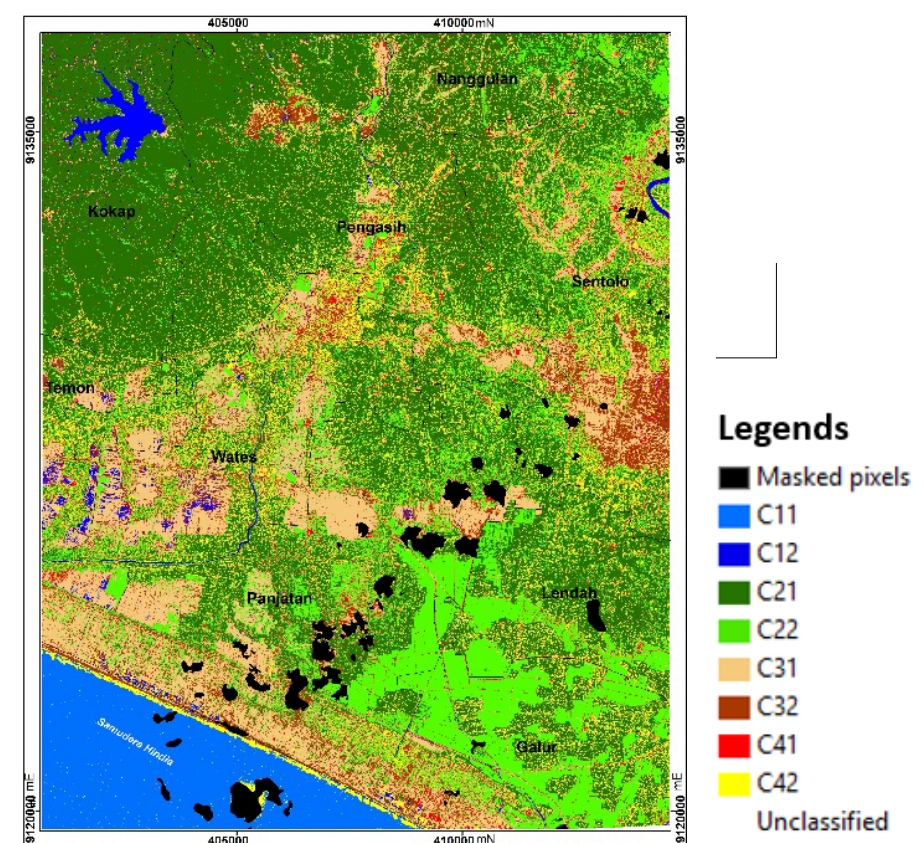

(b)

Figure 2. Spectral-related classification result using (a) Landsat-8 OLI (b) Pleiades. The class codes refer to Table 1.

Although produces relatively higher details as compared to Landsat-8 OLI result's, there were few objects could not be classified well as exhibited in Figure 1b, in comparison with those in Figure 1a. The higher details resulted from the high spatial resolution of Pleiades would possibly become a weakness to classify several classes of the homogeneous object in an extensive coverage, such as sea entitled as C11 and paddy field labeled as C22. As revealed in Figure 1b, several classification anomalies were found in both homogenous classes whereas few individuals or clustered pixels were classified into different class features amongst $\mathrm{C} 11$ or $\mathrm{C} 22$. Furthermore, the classification anomalies were also predominantly found in some features namely shadow, cloud or fog with the use of Pleiades imagery.

Although the relative radiometric correction and cloud masking were applied in the pre-processing stages, the bias which could decrease the resultant maps accuracies could not be significantly avoided using Pleiades. On the other hand, the various biases seem did not dominantly appear in the classification resulted by Landsat-8 OLI due to the use of three infrared bands which were resistant to some atmospheric disturbances. Whereas the appearance of the object's shadow (the shadow of the tree or building) did not appear in figure 1a because of the limited pixel size of Landsat- 8 OLI to be able recording the appearance of shadows which generally smaller than one pixel in its imagery. 


\subsection{Spatial-related land cover classification scheme}

In general, nine classes taken from spatial-related classification schemes could not be well classified using supervised classifications. It was clearly shown from the less representative appearance of the most classes resulting neither by Landsat-8 OLI (Figure 2a) nor Pleiades (Figure 2b) imagery. The fairly representative classification results were only found in the feature classes consisting of the water bodies (S11, S12, and S13) and vegetation class (S21). Even though there were some biases as found in the spectral-scheme classification results, the three classes consisting of water bodies were well-differentiated in the spatial-related classification scheme; thanks to infrared bands. The infrared bands contained in both imageries could differentiate S11, S12, and S13 classes based on its depth while S21 was identified by the density of its vegetation coverage.

In addition to several classes of objects, other feature classes in the spatial dimension cannot be distinguished properly using multispectral classifications as revealed by the low overall accuracy of the spatial-related land cover maps generated from both Pleiades and Landsat-8 OLI imageries. The low accuracy of both resulted maps as demonstrated in Table 2, was caused by the inability of these classification methods to accommodate the spatial features in the classification process. Therefore, other methods which could accommodate spatial features in the classification process are needed to produce higher accuracy maps for the spatial-related classification schemes.

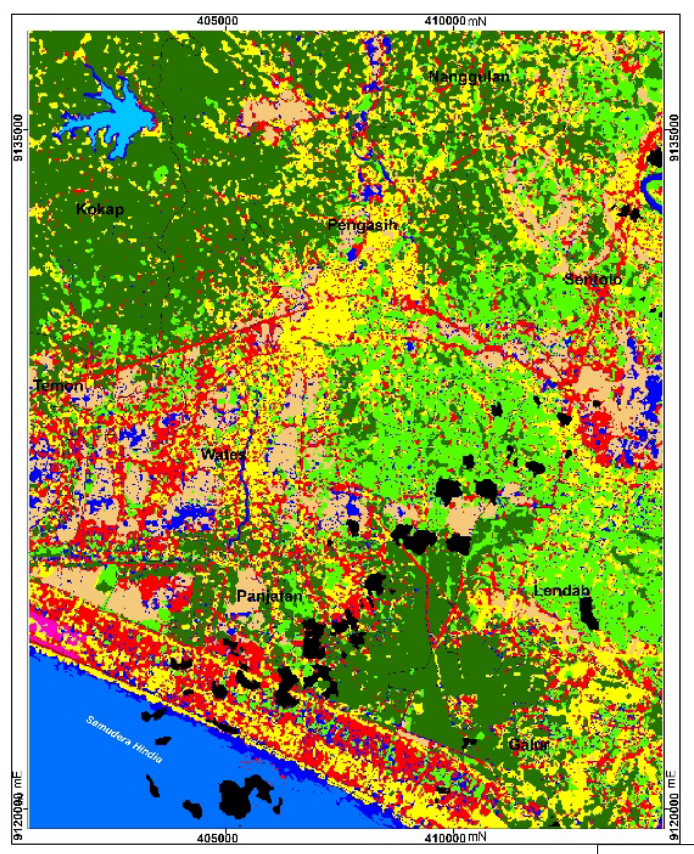

(a)

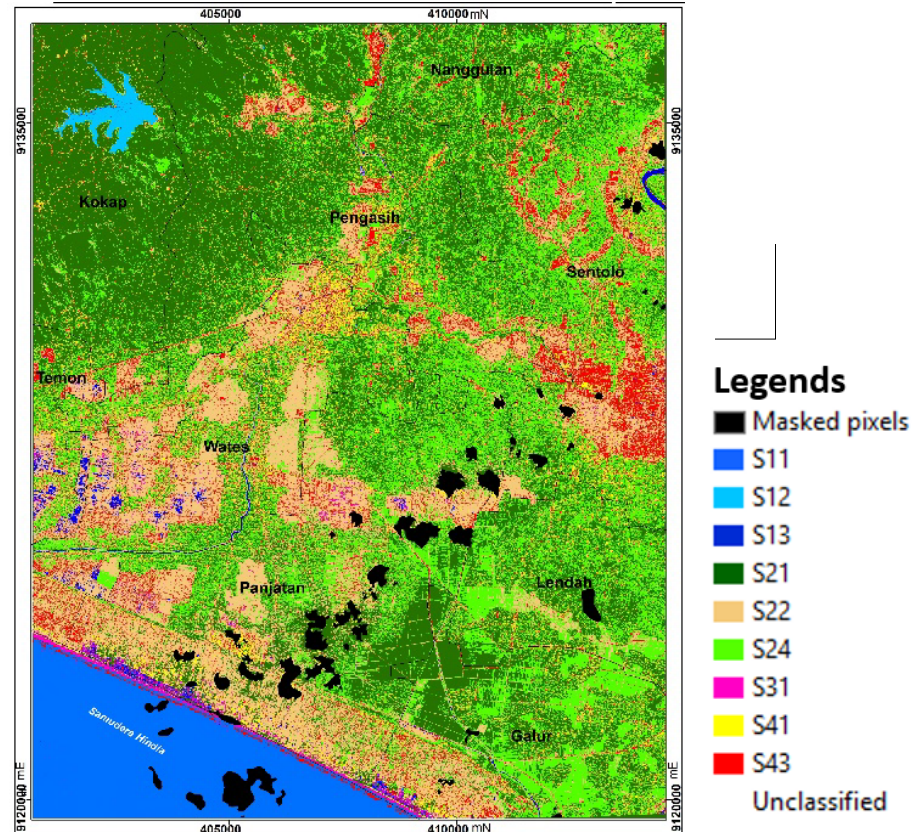

(b)

Figure 3. Spatial-related classification result using (a) Landsat-8 OLI (b) Pleiades. The class codes refer to the Table 1.

\subsection{Error matrix analysis}

Based on the error matrix analysis as listed in Table 2, it was known that the highest map accuracy gained from the usage of Pleiades for mapping the spectral-related land cover class. Although it has a lower spectral resolution rather than Landsat-8 OLI, the high spatial resolution of Pleiades could be elaborated to identify the object of interest more precisely. The small pixel size of Pleaides could minimize the tendency to take mixed pixel in the training area collection which attenuates the separability index as well as the classification accuracy. Besides, the presence of infrared bands as one of the four multispectral bands of Pleiades were considerably powerful enough to be used in distinguishing the spectral characteristics of the land cover classes in the spectral dimension. 
Table 2. Comparison of spectral and spatial-related land cover maps generated using Landsat-8 OLI and Pleiades

\begin{tabular}{|c|c|c|c|c|c|c|c|c|c|}
\hline \multirow{2}{*}{$\begin{array}{l}\text { Class } \\
\text { Code }\end{array}$} & \multicolumn{2}{|c|}{ Landsat-8 OLI } & \multicolumn{2}{|c|}{ Pleiades } & \multirow{2}{*}{$\begin{array}{l}\text { Class } \\
\text { Code }\end{array}$} & \multicolumn{2}{|c|}{ Landsat-8 OLI } & \multicolumn{2}{|c|}{ Pleiades } \\
\hline & $\begin{array}{c}\text { Producer } \\
\text { acc }\end{array}$ & $\begin{array}{l}\text { User } \\
\text { acc }\end{array}$ & $\begin{array}{c}\text { Producer } \\
\text { acc }\end{array}$ & $\begin{array}{c}\text { User } \\
\text { acc }\end{array}$ & & $\begin{array}{c}\text { Producer } \\
\text { acc }\end{array}$ & $\begin{array}{l}\text { User } \\
\text { acc }\end{array}$ & $\begin{array}{c}\text { Producer } \\
\text { acc }\end{array}$ & $\begin{array}{l}\text { User } \\
\text { acc }\end{array}$ \\
\hline $\mathrm{C} 11$ & 100 & 86,09 & 97,8 & 99,9 & S11 & 90,21 & 100 & 100 & 100,0 \\
\hline $\mathrm{C} 12$ & 84,02 & 71,50 & 91,6 & 81,7 & $\mathrm{~S} 12$ & 41,94 & 98,16 & 52,84 & 100,0 \\
\hline $\mathrm{C} 22$ & 63,49 & 80,27 & 68,8 & 94,4 & S13 & 76,56 & 17,79 & 31,40 & 73,6 \\
\hline $\mathrm{C} 22$ & 99,79 & 49,00 & 95,2 & 80,9 & S21 & 69,68 & 97,19 & 87,29 & 95,8 \\
\hline $\mathrm{C} 31$ & 36,60 & 91,78 & 69,2 & 66,3 & $\mathrm{~S} 22$ & 42,66 & 99,42 & 85,25 & 66,9 \\
\hline $\mathrm{C} 32$ & 100 & 30,81 & 59,7 & 51,7 & S24 & 12,86 & 3,01 & 55,39 & 6,2 \\
\hline $\mathrm{C} 41$ & 21,44 & 19,76 & 37,8 & 82,5 & S31 & 0,00 & 0,00 & 0,08 & 14,8 \\
\hline $\mathrm{C} 42$ & 15,81 & 61,08 & 77,7 & 24,4 & S41 & 95,15 & 80,15 & 81,57 & 61,9 \\
\hline \multicolumn{2}{|c|}{ Overall Accuracy } & 66,28 & & 81,7 & $\mathrm{~S} 43$ & 90,50 & 19,43 & 46,79 & 16,0 \\
\hline \multirow[t]{2}{*}{ Kappa } & & 0.59 & & 0,77 & \multicolumn{2}{|c|}{ Overall Accuracy } & 69,59 & & 64,6 \\
\hline & & & & & \multicolumn{2}{|c|}{ Kappa } & 0,65 & & 0,62 \\
\hline
\end{tabular}

However, the advantages of Pleaides usage were not relatable to be applied in spectral-related where the Pleiades gave the lowest overall accuracy value as compared to the other three classification results, even though the value was not significantly different from the usage of Landsat-8 OLI in this case. The low overall accuracies of the classification results using two images were due to the absence of spatial aspects that should be considered to produce spatial-related land cover maps. Therefore, the spatial-oriented classes such as S24 and S31 then gave the lowest user's and producer's accuracy which resulted in a decrease of overall accuracy as well as the Kappa coefficient in the classification results using both images.

\section{CONCLUSION}

This study revealed that the significant difference in spatial resolution resulting in the direct effects for the spectral-related classification results. With the usage of higher spatial resolution imagery, the classification process became more precise and it gave a better accuracy rather than its counterparts. However, the insignificant result of spatial-related land cover classes gained from the usage of different imageries, implicitly proved that multispectral classification is less suitable to generate spatial-related land cover maps.

\section{ACKNOWLEDGMENT}

This study was supported through a research grant funded by Indonesia Endowment Fund for Education as a part of the first author's master degree completion. Thanks also to the Indonesian's National Institute of Aeronautics and Space for providing the Pleiades imagery used in this study.

\section{REFERENCES}

[1] Weih, R. C., \& Riggan, N. D., "Object-Based Classification vs. Pixel-Based Classification: Comparative Importance of Multi-Resolution Imagery", The International Archives of the Photogrammetry, Remote Sensing and Spatial Information Sciences Vol XXVIII-4/C7, (2010).

[2] Estoque., C. R., Yuji, M., et al, "Pixel-based and Object-based Classifications using High and Medium Spatial Resolution Imageries in the Urban and Sub-Urban Landscapes", Geocarto International, 1113-1129, (2015).

[3] Danoedoro, P. "Developing a versatile land-use information System Based on Satellite Imagery for Local Planning in Indonesia Phase I: establishment of classification scheme", Proceedings, The $7^{\text {th }}$ International Seminar on GIS for Developing Countries (GISDECO-7), GIS Capacity Building and Infrastructure, Universiti Teknologi Malaysia, Skudai, Johor (2004) 
[4] Danoedoro, P., [Land-Use Information from The Satellite Imagery: Versatility and Contents for Local Physical Planning], USA, Lambert Academic Publishing, (2009).

[5] Jensen, J. R., [Introductory Digital Image Processing: A Remote Sensing Perspective 4th Edition], South Carolina, Pearson Education, (2016).

[6] Adam, H. E., et al., "A Comparison of Pixel-Based and Object-Based Approaches for Land Use Land Cover Classification in Semi-Arid Areas, Sudan", IOP Conference Series: Earth and Environmental Science, 1-10, (2016).

[7] Ozesmi, S. L., \& Bauer, M. E., "Satellite remote sensing of wetlands", Wetlands Ecology and Management, 381$402,(2002)$.

[8] Danoedoro, P., [Introduction to Digital Remote Sensing], Yogyakarta, Andi Publisher, (2012).

[9] Shalaby, A., \& Tateishi, R., "Remote sensing and GIS for mapping and monitoring land cover and land-use changes in the Northwestern coastal zone of Egypt", Applied Geography 27, 28-41, (2007).

[10]Lillesand, T. M., Kiefer, R. W., \& Chipman, J. W., [Remote Sensing and Image Interpretation: Sixth Edition], Madison, John Willey \& Sons, Inc., (2008).

[11] Lunetta, R. S., \& Knight, J. F., "An Experimental assessment of minimum mapping unit size", IEEE Transaction on Geoscience and Remote Sensing Volume 41. No.9, 2132-2134, (2003).

[12] Congalton, R. G., \& Green, K, [Assessing the accuracy of remotely sensed data: Principles and practices], Boca Raton, CRC Press, (2009). 